\title{
Die Polizei des Bundestagspräsidenten in parlamentsgeschichtlicher Perspektive
}

\author{
Ralph Igel und Michael F. Feldkamp*
}

Gemäß Art. 40 Abs. 2 Satz 1 Grundgesetz (GG) übt der Präsident des Bundestages das Hausrecht und die Polizeigewalt im Gebäude des Bundestages aus ${ }^{1}$. Zur Ausübung dieses Rechtes verfügt er über eine eigene Polizei. Grundsätzlich sind Polizeigewalt, Hausrecht und Disziplinargewalt des Bundestagspräsidenten zu unterscheiden.

\section{Polizeigewalt im Parlament - Hausrecht und Disziplinargewalt}

Disziplinargewalt ist nach Heinrich von Brentano di Tremezzo das innerhalb eines „Sonderkreises bestehende Gewaltverhältnis, kraft dessen eine Unterordnung des Einzelnen unter den Träger der Disziplinargewalt besteht, die bedingt ist durch die Angehörigkeit zu dem betreffenden Sonderkreis und durch den Austritt aus ihm jederzeit gelöst werden kann" ${ }^{2}$. Handeln die Angehörigen des Sonderkreises den in ihm geltenden Ordnungsbestimmungen zuwider, kann der Gewaltinhaber kraft seiner Disziplinargewalt gegen sie einschreiten. Die Verfassungen in der Bundesrepublik Deutschland überlassen die Regelungen der parlamentarischen Disziplinargewalt den Geschäftsordnungen (GO) der Parlamente. Damit sind die Parlamente selbst Träger der Disziplinargewalt und zu ihrer Ausübung berechtigt; in der Regel haben diese jedoch aus Gründen der Zweckmäßigkeit die Disziplinargewalt fast vollständig auf den Parlamentspräsidenten übertragen ${ }^{3}$. Um die Arbeit des Parlaments zu ge-

* Dem Beitrag liegt die unveröffentlichte Masterarbeit von Ralph Igel „Die Polizeigewalt des Parlamentspräsidenten - ist dafür eine eigene Bundestagspolizei erforderlich?“" zugrunde. Die Arbeit wurde im August 2010 an der Deutschen Hochschule der Polizei in Münster eingereicht und dort von Prof. Dr. Wolfgang Schulte betreut. Der hier vorliegende Beitrag wurde von Ralph Igel und Michael F. Feldkamp völlig neugefasst. Igel ist Angehöriger der Polizei beim Deutschen Bundestag und Feldkamp Mitarbeiter in der Bundestagsverwaltung. Sie geben hier aber ihre persönliche Meinung wieder. Grundlagen des Beitrags sind unter anderem: (1) Vereinbarung zur Wahrnehmung von Sicherungsaufgaben zum Schutz des Bundestages zwischen dem Präsidenten des Deutschen Bundestages, dem Innenminister des Landes Nordrhein-Westfalen und dem Bundesminister des Inneren vom 16. September 1975; (2) Vereinbarung zur Wahrnehmung von Polizei- und Sicherungsaufgaben zum Schutz des Reichstagsgebäudes in Berlin vom 30. März 1993; (3) Allgemeine Dienstanweisung für die Verwaltung des Deutschen Bundestages in der Fassung vom 7. Juni 2010; (4) Dienstanweisung für den Polizeivollzugsdienst beim Deutschen Bundestag in der Fassung vom 5. November 1993. Ferner konnten für den historischen Überblick die Unterlagen und Aufzeichnungen von Wilhelm Stödter sowie ein Gespräch mit ihm am 2. Mai 2010 hilfreich verwendet werden. Stödter war von 1972 bis 2002 Leiter der Polizei beim Deutschen Bundestag.

1 Art. 40 Abs. 2 Satz 1 GG: „Der Präsident übt das Hausrecht und die Polizeigewalt im Gebäude des Bundestages aus."

2 Heinrich von Brentano di Tremezzo, Die Rechtsstellung des Parlamentspräsidenten nach Deutschem Verfassungs- und Geschäftsordnungsrecht, Dissertation an der Universität Gießen 1930, S. 41.

3 Vgl. Ernst Rode, Die Polizeigewalt des Reichstagspräsidenten, Dissertation an der Universität Göttingen 1929, S. 13; Heinrich von Brentano di Tremezzo, a.a.O. (Fn. 2), S. 42. 
währleisten, erhält der Präsident Sanktions- und Einschreitmöglichkeiten ${ }^{4}$. Diese sind: Rüge, Ordnungsruf, Wortentziehung, Ausschluss ${ }^{5}$; zusätzlich wurde 2011 für den Deutschen Bundestag noch die Möglichkeit eines Ordnungsgeldes als Sanktion eingeführt ${ }^{6}$.

Das Hausrecht stammt aus dem Privatrecht und räumt dem Eigentümer Nutzungsrechte ein ${ }^{7}$. Das Hausrecht von Behörden (und auch das des Bundestagspräsidenten) ist hingegen öffentlich-rechtlicher Natur. Es dient dazu, die Funktionsfähigkeit des Parlaments wie der Verwaltung und damit die Erfüllung des Widmungszwecks der Sache zu gewährleisten ${ }^{8}$. Das Hausrecht des Bundestagspräsidenten besteht für alle Gebäude ${ }^{9}$, die der Bundestag für seine hoheitlichen Aufgaben nutzt. Art. 40 Abs. 2 Satz 1 GG räumt dem Präsidenten ein originär und substantiell zustehendes Hausrecht ein. Das Grundgesetz schränkt die Parlamentsautonomie ein, denn der Bundestag kann die Zuständigkeit für die Ausübung des Hausrechts nicht an sich ziehen oder gar in Ausübung des Hausrechts getroffene Entscheidungen des Präsidenten ändern oder aufheben ${ }^{10}$. Das Hausrecht des Präsidenten unterliegt allerdings auch gesetzlichen und rechtsstaatlichen Prinzipien und wird durch die Rechte anderer (etwa der Fraktionen, der Abgeordneten, der Mitglieder der Bundesregierung etc.) begrenzt. Die Mitglieder des Bundestages und die Fraktionen verfügen über ein eigenes Nutzungsrecht in den ihnen überlassenen Räumlichkeiten. Dies führt gleichwohl nicht dazu, dass die Fraktionen oder Abgeordneten völlig frei und außerhalb jeglicher rechtlichen Bindung über ihre Räume verfügen können; dies gilt zum Beispiel dann nicht, wenn sich Störungen oder Gefahren, die aus diesen Räumen hervorgehen, auf die Funktionen des Bundestages auswirken. In einem solchen Fall ist der Präsident als alleiniger Träger der Polizeigewalt und des Hausrechts in den Liegenschaften des Bundestages befugt, sein übergeordnetes Hausrecht durchzusetzen oder seine Polizeigewalt zur Abwehr dieser Gefahren beziehungsweise Beseitigung der Störung auszuüben ${ }^{11}$.

4 Vgl. Karl-Heinz Kleinschnittger, Die rechtliche Stellung des Bundestagspräsidenten, Dissertation an der Universität Münster 1963, S. 82.

5 Heinrich von Brentano di Tremezzo, a.a.O. (Fn. 2), S. 42.

6 Vgl. $\$ 37$ GOBT in der Fassung der Bekanntmachung vom 2. Juli 1980 (BGBl. I, S. 1237) zuletzt geändert durch Bekanntmachung vom 24. November 2011 (BGBl. I, S. 2454). Vgl. auch Oliver Borowy, Parlamentarisches Ordnungsgeld und Sitzungsausschluss: Verfassungsrechtliche Aspekte, in: ZParl, 43. Jg. (2012), H. 3, S. 635 - 657.

7 Vgl. Hans H. Klein, in: Theodor Maunz / Günter Dürig, Grundgesetz. Kommentar, Loseblattsammlung, München 2009, Art. 40, Rdnr. 139.

8 Vgl. ebenda, Art. 40, Rdnr. 140.

9 „Gebäude des Bundestages" umfasst alle der Verwaltung des Deutschen Bundestages unterstehenden Gebäude, Gebäudeteile und Grundstücke (\$ 7 Abs. 1 GOBT), also alle Räumlichkeiten, die dem Bundestag und seinen Einrichtungen zu dienen bestimmt sind, unabhängig davon, in wessen Eigentum sie sich befinden. Im Hinblick auf das Ziel des Art. 40 Abs. 2 GG, die Unabhängigkeit des Parlamentes zu sichern, sind als „Gebäude des Bundestages“ alle Gebäudeteile und Grundstücke anzusehen, die dem Deutschen Bundestag, seinen Fraktionen oder seiner Verwaltung zu dienen bestimmt sind ( $\$ 3$ Abs. 1 Dienstanweisung-PVD DBT; Hans H. Klein, a.a.O. (Fn. 7), Art. 40, Rdnr. 27). Werden Gebäude nur vorübergehend für Zwecke des Bundestages in Anspruch genommen, so beschränkt sich die Zuständigkeit auf die Dauer ihrer Inanspruchnahme; gleiches gilt für die Gebäude, die der Bundesversammlung oder ihrer Fraktionen zu dienen bestimmt sind. Vgl. Klaus Vogel / Wolfgang Martens, Gefahrenabwehr. Allgemeines Polizeirecht des Bundes und der Länder, Köln 1986, S. 72.

10 Vgl. Hans H. Klein, a.a.O. (Fn. 7), Art. 40, Rdnr. 146.

11 Vgl. ebenda, Art. 40, Rdnr. 167 f. 
Maßgeblich für die Bestimmung des Umfangs der Polizeigewalt des Bundestagspräsidenten ist der materielle Polizeibegriff ${ }^{12}$. Dem Präsidenten obliegen unstrittig alle präventivpolizeilichen Maßnahmen, die für die Abwehr einer Störung der öffentlichen Sicherheit und Ordnung im Gebäude des Bundestages erforderlich sind. Fraglich ist, in welchem Umfang neben der so genannten Sicherheitspolizei auch die Verwaltungspolizei ${ }^{13}$ zu subsumieren ist. Unklar beziehungsweise strittig ist daneben, ob dem Parlamentspräsidenten mit der Polizeigewalt auch die Kompetenz zur Strafverfolgung übertragen worden ist. Hier klafft eine große Lücke zwischen der „gelebten Wirklichkeit“, also der gängigen Praxis, sowie der älteren Literatur und den einschlägigen Gesetzeskommentaren. Zum einen werden durch die Polizei beim Bundestag jährlich circa 400 bis 500 Strafsachen ermittelt und bearbeitet bis zur Abgabe an die Staatsanwaltschaft; zum anderen hat sich der Polizeipräsident in Berlin für die Liegenschaften des Bundestag als „unzuständig erklärt"14. Die Polizei beim Bundestag wird hier täglich im Rahmen von Ermittlungsverfahren nach der Strafprozessordnung tätig, was bisher weder von der Staatsanwaltschaft noch von den letztendlich beteiligten Gerichten angezweifelt oder in Frage gestellt wurde. Als Polizeiherr eines zwar kleinen, aber verfassungsrechtlich besonders wichtigen örtlichen Bereiches, ist der Parlamentspräsident somit das „vornehmste Polizeiorgan der Bundesrepublik Deutschland“; er ist gewissermaßen „Polizeipräsident im Sprengel Bundestagsgebäude“15. Genau diese Rolle soll im weiteren näher dargestellt werden.

In Anlehnung an Art. 27 der Verfassung des Deutschen Reichs (1871) beziehungsweise Art. 26 und 28 der Weimarer Reichsverfassung (1919) entstand Art. 40 GG, ohne dass darüber auf dem Verfassungskonvent auf Herrenchiemsee (1948) oder im Parlamentarischen Rat in Bonn (1948/49) debattiert wurde ${ }^{16}$. Die im Grundgesetz dem Parlamentspräsidenten zugesprochene Polizeigewalt war längst selbstverständlich geworden. Bereits ein flüchtiger Blick in die Geschichte der deutschen Parlamente seit 1815 belegt, dass seit den frühkonstitutionellen Verfassungen die Zuerkennung einer Polizeigewalt an das Parlament beziehungsweise seinen Präsidenten zu finden ist ${ }^{17}$. Vorbilder waren die Parlamente in Frankreich und Belgien ${ }^{18}$. Die staatliche Exekutive sollte vor einem polizeilichen Zugriff gegenüber der

12 Vgl. Michael Köhler, Die Polizeigewalt des Parlamentspräsidenten im deutschen Staatsrecht, in: Deutsches Verwaltungsblatt, 107. Jg. (1992), S. 1577 - 1585, S. 1579. Der materielle Polizeibegriff befasst sich mit der Frage, was funktional unter Polizei als Verwaltungstätigkeit zu verstehen ist und unter welchen Voraussetzungen in die Rechtssphäre des Bürgers eingegriffen werden darf. Polizei im materiellen Sinn bezeichnet im Bund und in den Ländern einheitlich die Aufgabe der Abwehr von Gefahren für die öffentliche Sicherheit und Ordnung. Vgl. Klaus Vogel / Wolfgang Martens, a.a.O. (Fn. 9), S. 33.

13 Vgl. zu diesen Begriffen Klaus Vogel/ Wolfgang Martens, a.a.O. (Fn. 12), S. 48 f.

14 So in einer Mitteilung des Polizeipräsidenten von Berlin Hans-Dieter Glietsch im schriftlichen Interview am 25. Juni 2010.

15 Walter Jellinek, Verfassung und Verwaltung des Reichs und der Länder, in: Teubners Handbuch der Staats- und Wirtschaftskunde, Bd. 2, H. 2, Abt. 1: Staatskunde, Leipzig 1928, S. 72.

16 Vgl. dazu R. P. Dach, in: Bonner Kommentar zum Grundgesetz, Art. 40, 2 (150. Aktualisierung, Febr. 2011; Drittbearbeitung Brocker).

17 Vgl. dazu unter anderem Karl Spengler, Die rechtliche Stellung und die Befugnisse des Reichstagspräsidenten, Dissertation an der Universität Würzburg 1912; Fritz Gentemann, Die staatsrechtliche Stellung des Reichstagspräsidenten in Bezug auf die Disziplinargewalt, das Hausrecht und die Polizeigewalt, Dissertation an der Universität Göttingen 1927.

18 Vgl. Hans Reinecke, Die Polizeigewalt des Bundestagspräsidenten, Dissertation an der Universität Hamburg 1960, S. 152; Eduard Hubrich, Die parlamentarische Redefreiheit und Disciplin. Auf 
Legislative getrennt werden. Es hätte nicht gereicht, staatliche Polizeikräfte auszuschließen. Es bestand die Gefahr, dass ohne polizeiliche Sanktionierungsmöglichkeiten im Parlament ein „rechtsfreier Raum“ entstanden wäre ${ }^{19}$. Schließlich unterstreicht es die Souveränität des Parlaments, sich aus eigener Zuständigkeit heraus zu schützen.

\section{Ordnungsdienst - Hausinspektion - Polizeivollzugsdienst}

Die Ordnungs- und Sicherheitsaufgaben im Bundeshaus wurden in dem am 7. September 1949 konstituierten Deutschen Bundestag zunächst auf Anforderung des Bundestagspräsidenten Erich Köhler von Beamten der Kriminalpolizei Bonn wahrgenommen. Der Organisationsausschuss des Bundestages hatte erkannt, dass Polizeidienste unverzichtbar waren und deswegen schon in seiner Sitzung am 22. November 1949 empfohlen, „dass das Haus sich bemühen müsse, einen eigenen Sicherheitsdienst einzuführen“. Bis dahin sollten die im Bundestag tätigen Bonner Kriminalbeamten als „Hauspolizei“ bezeichnet werden. Aber schon im April 1950 richtete Köhler eine „Hausinspektion“ ein, deren Angehörige „Ordnungsaufgaben“"wahrzunehmen hatten. Die Dienststelle befand sich im Bundeshaus, der ehemaligen Pädagogischen Akademie in Bonn. Schon im Spätsommer des Jahres 1950 wurde die Hausinspektion in „Hausordnungsdienst“ (HOD) ${ }^{20}$ umbenannt. Seine Angehörigen erhielten eine grüne Armbinde mit der Aufschrift „Haus-Ordnungsdienst“ und wurden auch für die Aufgaben der Saaldiener im Plenarsaal herangezogen ${ }^{21}$.

Mit Erlass des Bundesministers des Innern (BMI) vom 30. August 1951 wurde im Einvernehmen mit dem Bundestagspräsidenten die Sicherungsgruppe Bonn des Bundeskriminalamtes (BKA) beauftragt, das Parlament zu schützen. Ihre Angehörigen nahmen polizeiliche Aufgaben wahr. Sie wurden dabei, wie zuvor die von ihnen abgelösten Kriminalbeamten des Polizeipräsidiums Bonn, vom HOD des Bundestages unterstützt. Dieser wurde nach und nach mit ausgebildeten Polizeibeamten aufgestockt und personell ausgebaut. $\mathrm{Zu}$ dieser Zeit traten die Beamten des HOD wiederholt an den Präsidenten mit der Bitte heran, die Rechtsstellung des HOD zu klären und ihn ebenso wie die eingesetzten Beamten der Sicherungsgruppe Bonn mit polizeilichen Aufgaben zu betrauen.

Am 4. März 1955 wurde in einer vorläufigen Dienstanweisung (DA) für den HOD die örtliche und sachliche Zuständigkeit festgelegt. Es hieß: „Der HOD hat im Rahmen seiner Befugnisse nach pflichtgemäßem Ermessen die notwendigen Maßnahmen zu treffen, um Gefahren für die Sicherheit und Ordnung in den der Verwaltung des Deutschen

der Grundlage von Rechtsvergleichung und Rechtsgeschichte dargestellt nach deutschem Recht, Berlin 1899, S. 430 f.; S. 439. Zur internationalen Entwicklung siehe jetzt auch den Beitrag von Tobias Kaiser, „Brachialgewalt [...] in der Kathedrale der nationalen Legislative“. Gewalt als Extrem parlamentarischer Kommunikation, in: Andreas Schulz / Andreas Wirsching (Hrsg.), Parlamentarische Kulturen in Europa. Das Parlament als Kommunikationsraum (Beiträge zur Geschichte des Parlamentarismus und der politischen Parteien, Bd. 162, Reihe: Parlamente in Europa I), Düsseldorf 2012, S. 195 - 226.

19 Vgl. Hans Reinecke, a.a.O. (Fn. 18), S. 154.

20 Eine Begründung dafür ließ sich in den ausgewerteten Akten nicht finden.

21 Vgl. Michael F. Feldkamp / Dirk Kunze, Mit Frack im Parlament. Ein Beitrag zur parlamentarischen Kultur und politischen Symbolik im Deutschen Bundestag, Baden-Baden 2009, S. 32. Hier auch eine Abbildung der Armbinde. 
Bundestages unterstehenden Gebäuden, Gebäudeteilen und Grundstücken abzuwenden. Der HOD besitzt keine Exekutivgewalt, sondern nur Befugnisse nach den gesetzlichen Bestimmungen über die Notwehr und den Hausfriedensbruch" 22 . Somit war klar gestellt, dass es sich beim HOD um keine Polizei im formellen und materiellen Sinne handelte, sondern um eine reine „Hauspolizei“. Die Angehörigen des HOD gehörten der Laufbahn des mittleren beziehungsweise gehobenen allgemeinen Verwaltungsdienstes an. Ihre Dienstbezeichnungen lauteten: Oberinspektor, Inspektor, Obersekretär, Sekretär und Assistent.

Bereits der Ordnungsdienst des Reichstags der Weimarer Zeit führte die Bezeichnung „Hauspolizei“ und hatte auch nur hausrechtliche Befugnisse ${ }^{23}$. Um dies hervorzuheben, wurde bei der ansonsten textgleichen Übernahme des $\$ 14$ Abs. 1 des Preußischen Polizeiverwaltungsgesetzes von 1931 im Abschnitt V der vorläufigen Dienstanweisung das Wort „öffentliche“ vor dem Wort „Sicherheit“ weggelassen. Ferner hatte der Direktor beim Deutschen Bundestag am 31. August 1955 verfügt, dass die Angehörigen des HOD die Waffe nur im Falle der Notwehr gebrauchen dürfen. Die Beamten waren damit zur Erfüllung ihrer Aufgaben lediglich mit den so genannten Jedermannsrechten ausgestattet. Bei Exekutivmaßnahmen waren mit der Genehmigung des Bundestagspräsidenten die Ordnungspolizei oder die Beamten der Sicherungsgruppe des BKA hinzuzuziehen.

Mit Ablauf des 30. April 1955 wurden auf Wunsch des Bundestagspräsidenten die Beamten der Sicherungsgruppe des BKA, die für den Schutz des Parlamentes abgestellt waren, vom BMI abgezogen und in die Verwaltung des Bundestages übernommen. Entsprechend wurden die Bestrebungen, eine eigene Sicherungs- beziehungsweise Polizeitruppe aufzustellen, vorangetrieben. Doch erst zum 31. Dezember 1960 wurde der HOD in das Bundespolizeibeamtengesetz (BPolBG) ${ }^{24}$ aufgenommen; in $\$ 1$ Abs. 2 BPolBG hieß es fortan: „Zu den Polizeivollzugsbeamten des Bundes gehören auch die Beamten des Ordnungsdienstes der Verwaltung des Deutschen Bundestages. "25 Damit wurde den Polizeivollzugsbeamten des HOD auch die mit Gesetz über den unmittelbaren $Z_{w a n g}{ }^{26}$ geregelten Befugnisse im Rahmen der Gefahrenabwehr und Strafverfolgung übertragen. Zwei Jahre später, am 30. November 1962, fanden diese Befugnisse auch Eingang in eine Allgemeine Verwaltungsvorschrift des Bundestagspräsidenten ${ }^{27}$. Danach konnten die Polizeivollzugsbeamten des HOD von Personen ein Handeln, Dulden oder Unterlassen verlangen und notfalls auch erzwingen.

22 Vgl. die vorläufige Dienstanweisung HOD, maschinenschriftlicher Umdruck, o.O. [Bonn] 1955, im Abschnitt V, Aufgaben.

23 Vgl. dazu Heinz Woldt, Die Polizeigewalt des Reichstagspräsidenten, Dissertation an der Universität Heidelberg 1934.

24 Gesetz zur Regelung der Rechtsverhältnisse der Polizeivollzugsbeamten des Bundes in der ursprünglichen Fassung vom 9. Juli 1960 (BGBl. I, S. 569), Kurztitel „Bundespolizeibeamtengesetz“.

25 Bundespolizeibeamtengesetz vom 3. Juni 1976 (BGBl. I, S. 1357), dort heißt es nun: „Dieses Gesetz gilt auch für die Polizeivollzugsbeamten beim Deutschen Bundestag."

26 Gesetz über den unmittelbaren Zwang bei Ausübung öffentlicher Gewalt durch Vollzugsbeamte des Bundes in der im Bundesgesetzblatt Teil III, Gliederungsnummer 201-5 veröffentlichten bereinigten Fassung, das zuletzt durch Artikel 28 der Verordnung vom 31. Oktober 2006 (BGBl. I, S. 2407) geändert worden ist.

27 Allgemeine Verwaltungsvorschrift des Präsidenten des Deutschen Bundestages zum Gesetz über den unmittelbaren Zwang bei Ausübung öffentlicher Gewalt durch Vollzugsbeamte des Bundes vom 30. November 1962. 
Ausgenommen waren jedoch die Bundestagsabgeordneten: „Die besondere Stellung der Mitglieder des Bundestages findet Berücksichtigung, indem die Anwendung unmittelbaren Zwanges gegen diese stets eine im Einzelfall bezogene besondere Anweisung des Präsidenten des Deutschen Bundestages voraussetzt. " 28

Lange vermied es die Bundestagsverwaltung, den Polizeivollzugsdienst beim Bundestag auch entsprechend zu benennen. Erstens verstand sich der Bundestag als ein „offenes Haus“ und Parlament des Volkes ${ }^{29}$, und zweitens schien die „innere Sicherheit“ Anfang der 1960er Jahre nicht gefährdet. So hielt die Verwaltung es für unangemessen, eine eigene Polizei zu halten. Auf Anordnung des Bundestagspräsidenten vom 19. Oktober 1964 wurde der HOD in „Hausinspektion beim Deutschen Bundestag“ umbenannt. Dem damaligen Leiter der Hausinspektion (ein Kriminalhauptkommissar) unterstanden sieben Kriminalbeamte im Bundeshaus in Bonn und vier Kriminalbeamte im Reichstagsgebäude in Berlin sowie der Aufsichts- und Ordnungsdienst und der Pfortendienst.

Am 10. November 1966 erließ Bundestagspräsident Eugen Gerstenmaier die Richtlinien über die Hausinspektion. Sie enthielten die einschlägigen Bestimmungen über Aufbau und Gliederung der Hausinspektion, deren Stellung und Rechtsgrundlagen sowie die Aufgaben des Ordnungsdienstes, des Streifen- und Pfortendienstes. Demnach unterstand der Ordnungsdienst ausschließlich den Weisungen des Bundestagspräsidenten. Zum ersten Male wurde die bisherige „Grauzone“ auf dem Gebiet der Rechtsgrundlagen für die Polizeivollzugsbeamten beim Deutschen Bundestag verlassen und ihnen erstmals verbindliche gesetzliche Vorschriften für ihr hoheitliches Handeln an die Hand gegeben. Die Richtlinien enthielten im Wesentlichen die Aufgabenzuweisung aus der vorläufigen Dienstanweisung vom 4. März 1955, berücksichtigten aber, dass die Beamten des Ordnungsdienstes zwischenzeitlich Vollzugsbeamtenstatus erhalten hatten. Die Kriminalbeamten des Ordnungsdienstes sollten im Rahmen ihrer Befugnisse als Vollzugsbeamte des Bundes handeln und nach pflichtgemäßem Ermessen Maßnahmen treffen, um Gefahren für die öffentliche Sicherheit und Ordnung im Bereich des Bundeshauses und im Reichstagsgebäude abzuwehren. Nur sie waren zur Feststellung beziehungsweise zur Festnahme eines Straftäters im Bundeshaus befugt. Die übrigen Beamten des Ordnungsdienstes ohne Vollzugsbeamtenstatus waren dazu nicht ermächtigt. Grundsätzlich blieb es weiterhin möglich, gegebenenfalls die Hilfe der Polizeibeamten des Landes Nordrhein-Westfalen im Rahmen der Amtshilfe gemäß Art. 35 GG in Anspruch zu nehmen.

\section{Polizeivollzugsdienste des Bundestages}

Rechtlich gesehen waren die Beamten des Ordnungsdienstes seit 1960 Polizeivollzugsbeamte des Bundes. Und obgleich sie beamtenrechtlich den Beamten des Bundeskriminalamtes, des Bundesgrenzschutzes (seit 2005 Bundespolizei) und den mit polizeilichen Aufgaben betreuten Beamten im Bundesministerium des Inneren gleichgestellt waren, zeichnete sich in den einzelnen Passagen der Richtlinien des Bundestagspräsidenten nur ein zaghafter Ansatz ab, den Vollzugsbeamten beim Bundestag auch exekutivpolizeiliche Aufgaben zu

28 Ebenda, Abschnitt III, Anordnung unmittelbaren Zwangs, Abs. 3, zu $\$ 7$.

29 So der ehemalige Leiter der Polizei beim Bundestag Wilhelm Stödter im Interview am 2. Mai 2010. 
übertragen. Der Vermerk eines leitenden Mitarbeiters der Hausinspektion belegt, wie sehr man auf öffentliches Ansehen bedacht war: „Vielleicht können die Abgeordneten an polizeilichen Vorschriften für die Ordnungskräfte im Hause Anstoß nehmen. Außerdem sei auch die Meinung der Presse zu berücksichtigen, welche die Einrichtung einer Polizei im Bundeshaus sicher nicht gutheißen würde. Es bleibt aber festzustellen, dass diese Denkweise, wenn sie denn bei einer Mehrheit existiert, ein für allemal der Vergangenheit angehört. Außerdem sollte auch nicht übersehen werden, dass es auch nur einige wenige oder sogar nur ein Pressevertreter war, der zur damaligen Zeit eine stets kritische Haltung zu den polizeilichen Ordnungskräften im Bundeshaus einnahm. "30

Im Jahre 1967 wurde die Hausinspektion wieder in „Ordnungsdienst“ umbenannt. Die Änderung stand im Zusammenhang mit der im März und April 1967 durchgeführten Prüfung der Bundestagsverwaltung durch den Bundesrechnungshof. Dieser hatte festgestellt, dass die Hausinspektion den Ordnungs-, Streifen- und Pförtnerdienst sowie die Botenmeister inklusive Reinigungsdienst umfasste. In seinem Gutachten empfahl der Rechnungshof, die Arbeitsgebiete zu trennen ${ }^{31}$. Doch schon im Zuge seiner 1969 angestoßenen Parlamentsreform wurde unter Bundestagspräsident Kai-Uwe von Hassel die Parlamentsverwaltung umgebaut und im Juli 1970 für die Ordnungsdienste wieder die Bezeichnung „Hausinspektion“ eingeführt. Sie wurde unterteilt in Ordnungsdienst, Streifendienst, Pfortendienst und Ausweisstelle. Die Dienststelle wurde von einem Kriminalhauptkommissar (Besoldungsgruppe A 11) geleitet, sein Vertreter war ein Kriminaloberkommissar (Besoldungsgruppe A 10). Weiter gehörten der Dienststelle zwölf Kriminalobermeister, vier Regierungssekretäre sowie 40 Streifenbeamte (Amts- und Oberamtsmeister) an.

\section{Ein Polizeigesetz für die Hausinspektion?}

Erst infolge des Terrors der Roten Armee Fraktion (RAF) wurde in den 1970er Jahren die Hausinspektion zu einer polizeilichen Vollzugsdienststelle des Bundes ausgebaut.

Der Bundestagspräsident, der Bundesminister des Innern und der Innenminister des Landes Nordrhein-Westfalen vereinbarten am 17. September 1975, zur Wahrnehmung von Sicherungsaufgaben am Bundeshaus den dortigen Polizeivollzugsdienst personell mit Polizeivollzugsbeamten des Bundesgrenzschutzes zu verstärken. In dieser Vereinbarung wurde unter anderem auch ein Sicherheitskonzept für die Bundestagsliegenschaften empfohlen und später auch haushaltsrechtlich umgesetzt. So wurden erst jetzt die Eingänge und die Pförtnerlogen durchgängig mit schusssicherem Panzerglas ausgestattet, das Gelände um das Bundeshaus weiträumig umzäunt, eine optische Überwachung von Sicherheitsbereichen mittels Kameras eingerichtet und das Personal der Hausinspektion durch 48 Polizeibeamte des Bundesgrenzschutzes (im Rahmen von beamtenrechtlichen Abordnungen) verstärkt.

Am 26. April 1976 wurde eine neue Dienstanweisung für die Hausinspektion in Kraft gesetzt. Erstmals erfolgte eine klare materiell-polizeiliche Aufgabenzuweisung an die Polizei-

30 Aus den Unterlagen des ehemaligen Leiters der Polizei beim Bundestag Wilhelm Stödter.

31 Der Präsident des Bundesrechnungshofes, Gutachten über die Organisation und Wirtschaftlichkeit der Verwaltung des Bundestages, Januar 1968, Anlage 10: Organisationsvorschläge zum Inneren Dienst und zum Bereich Technik, S. 8. 
vollzugsbeamten des Bundestages. Darüber hinaus wurden erstmals auch polizeiliche Einzelbefugnisse an die Beamten übertragen, unter anderem die Identitätsfeststellung, die Gewahrsamnahme von Personen, die Sicherstellung von Sachen, die polizeiliche Sicherstellung und Beschlagnahme, die Durchsuchung von Personen und Sachen. Ferner wurde in der Dienstanweisung ausdrücklich darauf hingewiesen, dass die Polizeivollzugsbeamten der Hausinspektion auch die Aufgaben zu erfüllen haben, die ihnen per Gesetz zugewiesen sind, darunter die Verfolgung von Straftaten und Ordnungswidrigkeiten nach den Vorschriften der Strafprozessordnung und des Ordnungswidrigkeitengesetzes.

1974 beschloss die Innenministerkonferenz das „Programm für Innere Sicherheit in der Bundesrepublik Deutschland" (PIS) mit dem Ziel, das materielle Polizeirecht und die Vorschriften über die Anwendung des unmittelbaren Zwanges zu harmonisieren. Der Musterentwurf für ein (bundes-)einheitliches Polizeigesetz hatte auch den Bundestagspräsidenten als Träger der Polizeigewalt berücksichtigt. Zwar wurde dieses Mustergesetz nie verabschiedet, doch wurde auf seiner Grundlage seit 1980 eine neue Dienstanweisung für die Hausinspektion erarbeitet. Der Entwurf wurde den parlamentarischen Gremien, dem Bundestagspräsidium sowie den Parlamentarischen Geschäftsführern und dem Bundesministerium des Innern zur Stellungnahme zugeleitet; dennoch wurde die Dienstanweisung nicht in Kraft gesetzt. Stattdessen ordnete Bundestagspräsident Richard Stücklen im September 1981 an, dass die Bundestagsverwaltung einen eigenen Entwurf für ein „Gesetz über den Polizeivollzugsdienst beim Deutschen Bundestag" erarbeiten solle. Ein solcher Entwurf des Wissenschaftlichen Dienstes des Bundestages wurde am 13. August 1982 dem Bundesminister des Innern und dem Bundesminister für Justiz zur Stellungnahme übersandt.

Das Bundesministerium des Inneren teilte am 5. April 1984 (Az.: P II 5 - 640 143/1) dem Vorsitzenden des Innenausschusses Axel Wernitz (SPD) mit, dass es auch ohne ein normiertes polizeiliches Befugnisgesetz keine „verfassungsrechtlichen Bedenken gegen das polizeiliche Tätigwerden der Beamten der Hausinspektion “ gebe. Entsprechend hatte der Bundesminister des Innern, Friedrich Zimmermann (CSU), am 9. November 1983 im Innenausschuss erklärt, es stünde doch „,anderen Polizeien des Bundes“ - und damit eben auch der Hausinspektion beim Deutschen Bundestag - „offen, das Gesetz über den Bundesgrenzschutz für anwendbar zu erklären, wie dies bereits im BKA-Gesetz für den Schutz- und Begleitdienst (Personenschutz) geschehen" sei $^{32}$.

Der Bundesminister für Justiz (BMJ) teilte am 27. Dezember 1983 (Az.: 4006/1 - 6 - 44 289/83) dem Bundestagspräsidenten mit: „Sofern die Beamten des Polizeivollzugsdienstes des Deutschen Bundestages spezifische Grundrechtseingriffe vornehmen, kann nach meiner Auffassung bei der geltenden Rechtslage im Ergebnis ein verfassungsrechtliches Risiko nicht ausgeschlossen werden." Es schien also aus Sicht des BMJ sehr wohl nötig, ein eigenes Gesetz für die Polizeivollzugsbeamten des Bundestages zu erlassen, um eine verfassungskonforme Grundlage zu schaffen. Entsprechend wurde in der Folge der Wissenschaftliche Dienst vom Bundestagspräsidenten erneut beauftragt, einen Entwurf für ein „Gesetz über den Polizeivollzugsdienst beim Deutschen Bundestag“ zu erarbeiten. Am 3. Oktober 1984 lag dieser Entwurf vor. Nach dem Vorbild des BKA-Gesetzes ${ }^{33}$ wurden die Befugnisregelungen des

32 Protokoll der Sitzung des Innenausschusses vom 9. November 1983, Anlage 2, S. 13/8-9.

33 Gesetz über das Bundeskriminalamt und die Zusammenarbeit des Bundes und der Länder in kriminalpolizeilichen Angelegenheiten in der ursprünglichen Fassung vom 8. März 1951 (BGBl. I, S. 165); zuletzt geändert mit Gesetz am 25. Dezember 2008 (BGBl. I, S. 3083). 
Bundesgrenzschutzgesetzes für anwendbar erklärt; außerdem sollte der Name der Dienststelle von „Hausinspektion“ in „Polizeivollzugsdienst beim Deutschen Bundestag“ geändert werden.

Schließlich enthielt der Entwurf im Interesse der Einheitlichkeit eine Klausel, um auch in Berlin Gültigkeit zu haben. Hier wäre auch das damals noch geltende Viermächteabkommen über Berlin vom 3. September $1971^{34} \mathrm{zu}$ beachten gewesen. Das Polizeigesetz hätte auch für die Beamten der Bundestagspolizei im Referat PBl 4 im Berliner Reichstagsgebäude Wirkung entfaltet; daher wäre zwingend die Zustimmung aller Alliierten Stadtkommandanten erforderlich gewesen, was ein hohes Maß an Verhandlungen, Gesprächen und Bürokratie nach sich gezogen hätte. Dies alles zusammen führte letztendlich dazu, dass sich der Bundestagspräsident am Ende doch der Auffassung des Bundesinnenministers anschloss, wonach Art. 40 Abs. 2 GG als allgemeine „Generalklausel“ für das polizeiliche Tätigwerden der Polizeibeamten des Bundestages ausreichend sei. Der vom Wissenschaftlichen Dienst des Bundestages erarbeitete Gesetzentwurf für den Polizeivollzugsdienst wurde nie eingebracht.

\section{Der Polizei- und Sicherungsdienst des Deutschen Bundestages}

Erst 1989 wurde von Bundestagspräsidentin Rita Süssmuth die Hausinspektion in „Polizeiund Sicherungsdienst“ beim Deutschen Bundestag umbenannt, um schließlich auch den Polizeicharakter der Dienststelle im Namen herauszustellen. Die neu erarbeitete Dienstanweisung (basierend auf dem Musterentwurf eines einheitlichen Polizeigesetzes) zur Konkretisierung des Art. 40 Abs. 2 Satz 1 GG wurde nach Zustimmung aller parlamentarischen Gremien, der Fraktionen und des Präsidiums von Bundestagspräsidentin Rita Süssmuth am 5. November 1993 in Kraft gesetzt.

Insbesondere mit der Umbenennung der Dienststelle in „Polizei- und Sicherungsdienst“ wurde der Tätigkeit der längst bestehenden Bundestagspolizei auch von den Polizeibehörden der Länder und des Bundes der angemessene Respekt als Polizeibehörde entgegengebracht. Oft wurden zuvor die Zuständigkeiten sowie der Status als Polizeibehörde in der täglichen Zusammenarbeit von anderen Polizeibehörden angezweifelt oder sogar negiert. Dies führte nicht selten auch zu Verzögerungen bei der Wahrnehmung polizeilicher Aufgaben.

Spürbar verbesserte sich daraufhin die Akzeptanz bei der Landespolizei Nordrhein-Westfalen, dem Bundeskriminalamt und dem Bundesgrenzschutz, die für die Sicherungsmaßnahmen der direkt angrenzenden Regierungsbehörden wie Bundeskanzleramt und Bundesministerien zuständig waren. Seither funktionierte die Zusammenarbeit zumeist „geräuschlos“.

34 Viermächte-Abkommen zwischen den USA, der UdSSR, Großbritannien und Frankreich vom 3. September 1971, Abdruck in deutscher Übersetzung, in: Bundesministerium für innerdeutsche Beziehungen (Hrsg.), Zehn Jahre Deutschlandpolitik, Bonn 1980, S. 158 ff.; S. 188 f. (Schlussprotokoll). 


\section{Formen der Zusammenarbeit}

Die gute und arbeitsteilige Zusammenarbeit mit anderen Polizeikräften des Landes und des Bundes war und ist in den letzten Jahrzehnten insbesondere bei „Großeinsätzen und besonderen Lagen“ erforderlich. Beispielhaft seien hier der Weltwirtschaftsgipfel in Bonn (1978), Großdemonstrationen gegen die Nachrüstung während des „Kalten Krieges“ (1981) und schließlich Besuche von zum Teil hochgefährdeten ausländischen Staatsoberhäuptern oder Regierungschefs (Israel, USA, Russland, Vatikan etc.) genannt ${ }^{35}$. Bei ausländischen Staatsgästen, die vor dem Deutschen Bundestag sprachen oder diesen besuchten, war zu berücksichtigen, dass zum Beispiel im Bereich des Plenarsaals keine Waffen getragen werden dürfen, es sei denn, dass der Bundestagspräsident dies den Personenschützern ausdrücklich erlaubt. Entsprechend waren und sind bei der Vorbereitung derartiger Staatsbesuche Abstimmungen zwischen allen beteiligten Polizei- und Sicherheitskräften erforderlich, vor allem bei der Justierung und Absprache der Zuständigkeiten, des Verkehrs- und Vorfahrtskonzeptes, von Maßnahmen des Schutzkonzeptes allgemein, des (Sonder-)Ausweisregimes sowie individueller Personenschutzmaßnahmen (insbesondere den ausländischen Personenschutz betreffend). Darüber hinaus findet ständig eine enge Abstimmung mit dem Landeskriminalamt, der Polizeidirektion 3 und dem Bundeskriminalamt im Hinblick auf die allgemeine Sicherheitslage und die Gefährdungsbewertung der Bundestagsliegenschaften statt. Als im November 2010 Warnhinweise des Bundesinnenministers ergingen, wurde das Reichstagsgebäude vom Landeskriminalamt in die Gefährdungsstufe 2 eingestuft. Daraufhin wurde mit der vorgelagerten Besucherkontrolle in der Containeranlage auf dem Platz der Republik eine neue Zugangsregelung für Besucher getroffen.

\section{Die Polizei und Sicherungsdienste innerhalb der Bundestagsverwaltung}

Die Polizei gehört zu einer Organisationseinheit der Bundestagsverwaltung. Leiter der Verwaltung ist der Präsident des Bundestages. Seine Aufgaben nimmt im Auftrage der Direktor beim Deutschen Bundestag (im Range eines Staatssekretärs) wahr. Die Polizei und die Sicherungsdienste sind organisatorisch als „Referat ZR 3 “ in die Bundestagsverwaltung eingegliedert. Die Buchstaben- und Zahlenkombination weist auf das dritte von vier Referaten innerhalb der „Unterabteilung ZR (Recht)“ hin, die wiederum zur „Abteilung Z (Zentralabteilung)“ gehört. Das Referat führt die Bezeichnung „Polizei, Sicherungsaufgaben“. Der Unterabteilung ZR sind des Weiteren die Referate für Geheimschutz, Justitiariat und Haushalt zugeordnet.

Aufgrund dieser Organisation sind die Polizeibeamten des Bundestages verwaltungstechnisch nicht direkt dem Bundestagspräsidenten unterstellt. Die Vorgesetzten sind auf den jeweiligen Hierarchieebenen weisungsbefugt gegenüber den Polizeikräften. Weder kann sich die Bundestagspolizei direkt an den Präsidenten wenden, noch hat umgekehrt der Präsident einen „direkten Draht“ zu „seiner Polizei“. In der Praxis ist eine Einflussnahme durch die

35 Beispiele bei Michael F. Feldkamp / Dirk Kunze, a.a.O. (Fn. 21), S. 24; Hélène Miard-Delacroix, Reden ausländischer Gäste im Parlament am Beispiel des Deutschen Bundestages, in: Andreas Schulz / Andreas Wirsching (Hrsg.), a.a.O. (Fn. 18), S. 177 - 193, S. 182 f. 
zwischengeschalteten Verwaltungsvorgesetzten möglich. Einerseits bekämen die Polizeikräfte des Bundestages möglicherweise einen anderen Stellenwert, wenn sie wie das Protokoll beim Direktor oder wie das Pressezentrum direkt beim Präsidenten als Stabsstelle angegliedert wären. Andererseits kann man sicherlich geltend machen, dass mit der Aufwertung der Polizeikräfte ihre doch größtenteils Routinearbeit im Hinblick auf die Aufgaben des Bundestages eine unangemessene Überbewertung erhielte.

Dennoch darf nicht übersehen werden, dass das Grundgesetz dem Bundestagspräsidenten die Polizeigewalt direkt übertragen hat. Es handelt sich insoweit um eine ihm selbst zustehende, vom Bundestag unabhängig wahrzunehmende Kompetenz ${ }^{36}$.

\section{Dienststelle mit Zukunft}

Im Laufe der Geschichte des Bundestages erfolgte die Ausübung der Polizeigewalt des Bundestagspräsidenten entweder durch eigene Polizei- und Ordnungskräfte oder durch im Einzelfall unterstellte Polizeikräfte der „ordentlichen Polizei“ (Landespolizei). Der Bundestag hatte bei dieser Praxis an die Tradition der Kaiserzeit und der Weimarer Zeit angeknüpft, sich aber im Laufe der Jahrzehnte zunehmend eigener Sicherheits- und Ordnungskräfte bedient, aus denen sich schließlich die Polizei beim Deutschen Bundestag entwickelte.

Dieser Prozess wurde mutig und konsequent insbesondere durch die Bundestagspräsidentin Rita Süssmuth vorangetrieben. Mit dem Umzug des Bundestages von Bonn nach Berlin wurde die Entwicklung von der Hausinspektion zur Polizei formal vollzogen. Bei der täglichen Arbeit entstehen sowohl in rechtlicher, organisatorischer als auch personeller Hinsicht immer wieder neue Bedingungen, die es erfordern, die Dienststelle weiter anzupassen und zukunftssicher auszubauen.

Ein Desiderat ist es, die rechtlichen Rahmenbedingungen mit der gelebten Wirklichkeit und täglichen Dienstausübung in Einklang zu bringen und eine Novellierung und Anpassung der Absprachen zu treffen. Die Polizei beim Deutschen Bundestag aber leistet auch so täglich ihren Beitrag für die Sicherheit der Abgeordneten und deren Mitarbeiter sowie für die Gäste und Besucher des Parlaments und ist somit eingebettet in das Gefüge der Sicherheitsorgane der Bundesrepublik Deutschland. Nicht ohne Grund stellte Bundestagspräsident Norbert Lammert fest: „Für die Polizei beim Deutschen Bundestag gilt in besonderer Weise, was für die Verwaltung im Allgemeinen zutrifft: je unauffälliger sie arbeitet, desto besser ist sie." 37

36 Vgl. Hans H. Klein, a.a.O. (Fn. 7), Art. 40 GG, Rdnr. 147; Michael Köhler, a.a.O. (Fn. 12), S. 1577, S. 1584; BVerfGE 108, S. 251, S. 273.

37 Bundestagspräsident Prof. Dr. Norbert Lammert im schriftlichen Interview vom 26. April 2010. 